\title{
Circadian pattern in occurrence of renal colic in an emergency department: analysis of patients' notes
}

Roberto Manfredini, Massimo Gallerani, Olga la Cecilia, Benedetta Boari, Carmelo Fersini, Francesco Portaluppi

According to anecdotal evidence people are more at risk of renal colic during the night. Although this has never been investigated, several studies have shown a circadian variation for other acute diseases. ${ }^{1}$ We investigated whether renal colic occurred in a circadian pattern.

\section{Methods and results}

We reviewed all episodes of renal colic from 1 January 1990 to 31 December 1996 in the emergency department of St Anna Hospital, which serves the 150000 people in the city and suburbs of Ferrara, Italy. Altogether 3410 episodes of renal colic were reported $(66.0 \%(2281)$ in men). The mean age of the patients was 46 years (SD 16 years). The time (within 30 minutes) when symptoms started could be determined for $3360(98.5 \%)$ patients (2272 men and 1088 women). All patients were physically examined and had radiological, ultrasound, urine, and blood tests. Kidney stones were found in 1641 patients (48.8\%; 1134 men and 507 women), of which $71.0 \%$ (1165) were calcium stones. For the main statistical analysis of the 3360 patients for whom time of onset could be determined precisely, we used a partial Fourier series with up to four harmonics $(24,12,8,6$ hours), categorising each precise time of event as one of 24 increments of one hour. To test goodness of fit we calculated the "percentage of rhythm," the percentage of overall variability of data about the arithmetic mean attributable to the fitted rhythmic function. We used the $\mathrm{F}$ test to determine the significance of the fit of each function.

The 50 patients for whom the onset time of the episode of renal colic could not be determined precisely had the same confounding factors as the other 3360-age, sex, race, and underlying condition of nephrolithiasis. A secondary analysis including these 50 patients showed that excluding them from the main analysis did not significantly affect the results.

A highly significant $(\mathrm{P}<0.001)$ circadian rhythm was found, with a morning peak and an afternoon low (figure) both in the population as a whole (overall peak 0432 hours (95\% confidence interval 0320 to 0556 ), percentage of rhythm $62.8 \%$ ), and in subgroups by sex (men: $\mathrm{n}=2272,0456$ (0328 to 0624), 56.6\%; women: $\mathrm{n}=1088,0411$ (0300 to 0520$), 66.7 \%)$. Participants with or without kidney stones did not differ $(\mathrm{n}=1641,0448$ (0315 to 0550 ), 59.2\%; $\mathrm{n}=1719,0424$ (0305 to 0535 ), $64.4 \%$, respectively).

\section{Comment}

Episodes of renal colic show a significant circadian pattern, with a morning peak and a minimum in the afternoon. Urine production and renal excretion rates of solutes rise during daytime and reach minimum values

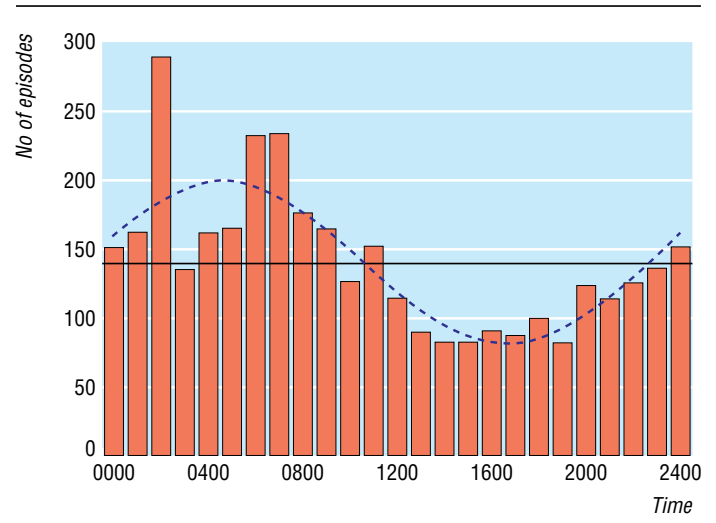

Episodes of renal colic in 3360 patients at different times of day

at night. In healthy people, the glomerular filtration rate shows a circadian rhythm peaking in the morning, with a relative amplitude of $33 \%{ }^{2}$ This rhythm means that urine is hyperconcentrated during night time, which entails a risk of nephrolithiasis and infections. The lithogenic risk for calcium oxalate stones is highest at the end of the night or during the early morning, when urinary output is minimal. ${ }^{3}$ Studies on healthy people and people who have had kidney stones showed a higher risk of calcium oxalate crystallisation in the morning. ${ }^{4}$ The inhibitory activity of calcium oxalate crystallisation showed a marked circadian rhythm in both groups, but in people who had had kidney stones the peak time was delayed (0900-1000 v 0500-0600). A circadian variation with a midnight peak, independent of meal times, has been reported for episodes of biliary colic. ${ }^{5}$

Contributors: RM, MG, and FP initiated and developed the primary study hypothesis, discussed the core ideas, designed the protocol, and participated in the collection and analysis of data and writing the paper. $\mathrm{OlC}$ and $\mathrm{BB}$ discussed core ideas and participated in the study design, analysis, and interpretation of data, and in writing the paper. CF developed the primary study hypothesis, participated in interpretation of the data, quality control, and writing the paper. RM and FP are the guarantors.

Funding: University of Ferrara.

Competing interests: None declared.

1 Portaluppi F, Manfredini R, Fersini C. From a static to a dynamic concept of risk: the circadian epidemiology of cardiovascular events. Chronobiol Int 1999;16:33-49.

2 Koopman MG, Koomen GC, Krediet RT, de Moor EA, Hoek FJ, Arisz L. Circadian rhythm of glomerular filtration rate in normal individuals. Clin Sci 1989;77:105-11.

3 Robert M, Roux JO, Bourelly F, Boularan AM, Guiter J, Monnier L. Circadian variations in the risk of urinary calcium oxalate stone formation. $\mathrm{Br}$ J Urol 1994;74:294-7.

4 Singh RK, Bansal A, Bansal SK, Singh AK, Mahdi AA. Circadian periodicity of urinary inhibitor of calcium oxalate crystallization in periodicity of urinary inhibitor of calcium oxalate crystallization
healthy Indians and renal stone formers. Eur Urol 1993;24:387-92.

5 Rigas B, Torosis J, McDougall CJ, Vener KJ, Spiro HM. The circadian rhythm of biliary colic. J Clin Gastroenterol 1990;12:409-14.

(Accepted 1 November 2001)
First Internal

Medicine,

Department of

Clinical and

Experimental

Medicine,

University of

Ferrara Medical

School, via

Savonarola 9,

I-44100 Ferrara,

Italy

Roberto Manfredini

assistant professor of

internal medicine

Olga la Cecilia

resident, school of

specialisation in

nephrology

Benedetta Boari

resident, school of

specialisation in

internal medicine

Carmelo Fersini

professor of internal

medicine

Francesco

Portaluppi

professor of medical

therapy

Second Internal

Medicine,

Department of

Clinical and

Experimenta

Medicine,

University of

Ferrara Medical

School

Massimo Gallerani

medical assistant

Correspondence to:

R Manfredini

mfr@unife.it

BMJ 2002;324:767 